\title{
Real time assessment of hand-arm vibration system based on capacitive MEMS accelerometers
}

\author{
Giuseppe Aiello $^{\mathrm{a}}$, Giada La Scalia ${ }^{\mathrm{a}, *}$, Mariangela Vallone $^{\mathrm{b}}$, Pietro Catania $^{\mathrm{b}}$, Mario Venticinque ${ }^{\mathrm{c}}$ \\ a Dipartimento di Ingegneria Chimica, Informatica, Gestionale, Meccanica, Università di Palermo, Viale delle scienze Ed. 8, 90128 Palermo, Italy \\ ${ }^{\mathrm{b}}$ Dipartimento dei Sistemi Agro-Ambientali (SAGA), Università di Palermo, Viale delle Scienze Ed. 4, 90128 Palermo, Italy \\ ${ }^{c}$ Agroindustry Advanced Technologies S.p.A., Blocco Palma I, Zona Industriale, 95121 Catania, Italy
}

\section{A R T I C L E I N F O}

\section{Article history:}

Received 12 September 2011

Received in revised form 27 February 2012

Accepted 28 February 2012

\section{Keywords:}

Hand-arm vibration

MEMS acceleration-sensor

Wireless sensing technology

Health risk assessment

\begin{abstract}
A B S T R A C T
Vibrations are a well known potential cause of health diseases and therefore constitute a main concern for the safety of workers in a large number of activities. In order to prevent health hazards, national and international institutions have issued laws and directives which establish recommended limits to the workers' exposure to vibrations during operations. Consequently, if the amount of adsorbed vibration exceeds the allowable daily limits the worker has to stop his job. Recent wireless sensing and communicating technologies can effectively be employed for such purpose, allowing to develop monitoring customized devices at affordable cost which could be easily employed during the workers' activity. In such context, the present research proposes an innovative system aimed at estimating the hand-arm exposure to vibration according to the Standard EN ISO 5349-1:2004. In particular the proposed system is based on Micro Electro-Mechanical Systems (MEMS) technology and involves the design of a compact wearable unit to be attached to the waist of the operator and a fixed station for data storage and analysis. The paper reports the design of such a system and an experimental test performed on hand held shakers commonly employed for olive harvesting. The results highlight the opportunities offered by these emerging technologies in developing intelligent devices to be included in the worker's equipment which might significantly modify the current approach to safety management in the next future.
\end{abstract}

(c) 2012 Elsevier B.V. All rights reserved.

\section{Introduction}

Mechanization in agriculture is a key driver of productivity and competitiveness, particularly for industrialized countries with high labour cost. The use of tools and machines throughout the entire supply chain from land development to harvesting, storage, and processing has therefore become, nowadays, a standard practice in almost all agro-industrial productions. Unfortunately, the increase in the mechanization level has introduced additional sources of risk for operators, such as noise, vibrations, etc., thus affecting the sustainability of the sector.

In olive oil production, for example, where poor harvesting efficiency is probably one of the worst hidden costs, mechanized techniques have lead to significant efficiency increase. In such context, hand held harvesting units detaching the drupe through vibration are frequently used, and the workers are consequently exposed to high levels of vibrations. It is nowadays well recognized that such exposure to vibrations is a potential cause of muscular/skeletal

\footnotetext{
* Corresponding author.

E-mail addresses: aiello@dtpm.unipa.it (G. Aiello), g.lascalia@leonardoengineering. net (G. La Scalia), m.vallone@unipa.it (M. Vallone), mventicinque@aatspa.it (M. Venticinque).
}

pains in hand-arm system, and specific pathologies such as Vibration-Induced White Finger (VWF) and Carpal Tunnel Syndrome (CTS), Hand-Arm Vibration Syndrome (HAVS) (Gerhardsson et al., 2005).

Due to the potential hazardousness of such shakers, they are generally designed with the aim of transmitting maximum vibration to the branches in order to optimize fruit detachment while minimizing vibration transmission to the operator (Horváth and Sitkei, 2001; Gil et al., 2001). Although the variety of designs for vibrating heads are wide ranging, all vibrators currently used in olive-tree harvesting involve a kinematic mechanism to transfer the motion coming from a motor to a shaft or to an inertial-mass, thus generating a periodic propelling force. Typically several methods and means are suggested in order to minimize the vibration absorption for the worker, for example the use of anti-vibration gloves, and advanced vibration damping devices designed to reduce the vibration level on the handle. Nevertheless these practices represent simple palliatives, because not only the vibration intensity but also the exposure time is an important risk factor and even a prolonged exposure to reduced levels of vibration can be hazardous for the workers. In order to prevent possible health hazards, therefore, national and international governmental institutions have issued laws and directives to fix recommended limits 
for vibration exposure. According to such recommendations the total exposure has to be estimated as the sum of several cumulative contributions of vibration during the daily worker activity, then, if the amount of adsorbed vibration exceeds the allowable daily limits, the worker has to stop his job. The maximum allowable operating time is commonly calculated on a reference vibration value, generally provided by the manufacturer. This approach is however questionable as in fact the effective vibration intensity and the hand-arm system biodynamic response to mechanical vibrating machines largely depend upon several context-specific factors such as maintenance or operating conditions, vibration direction, frequency, posture, grip force, operating tool, handle sizes, etc. (Burström, 1997; Aldien et al., 2006; Dewangan and Tewari, 2008). A more effective approach to reduce the health risks originated by vibrations, would rather involve to equip the worker with suitable and reliable instruments to monitor and analyze the vibration exposure, thus providing a valuable information to prevent health hazards. The practicability of this approach requires to equip the worker with suitable and reliable instruments to estimate and analyze the vibration exposure in real operating conditions. This is not always easy depending on the operating context, as in the case of agricultural applications, where activities are generally performed outdoor in adverse climatic conditions and without reliable power supply.

This research addresses this topic by proposing an innovative system for outdoor vibration measurement and analysis coherently to the Standard ISO 5349 and based on recent technologies, nowadays available on the market at affordable cost. The research involves the definition of the hardware components of the system and the related software development activities specifically referred to the applicative scenario of the harvesting operations, as well as an experimental test in real operating conditions. The experiments have been carried out on olive shaking tools commonly employed in olive harvesting operations and allowed to gather and characterize the vibration data, in terms of frequency spectrum and intensity. Such information has been subsequently exploited for real-time vibration monitoring and health hazard assessment.

The aim of the study is to demonstrate how new technologies can be effectively employed to gather relevant data on workers' risk exposure, even in the adverse environmental conditions which frequently occur in agro-industrial activities. This information can be valuable for a more responsible approach towards the management of agro-industrial activities, suggesting for example future scenarios where properly designed decision support systems may be employed in the scheduling of workers' shifts or the maintenance operations.

\section{Materials and methods}

The research steps performed to develop the wireless vibration sensing device here proposed involved a preliminary characterization of the specific operating context considered, which lead to the definition of the operating requirements. As stated before, the target operating context considered is that of the mechanized olive harvesting operations, carried out by means of hand-held shaking tools. A olive shaker has hence been individuated among those commonly employed by olive harvesters. Such machine has been preliminary analyzed with the aim of characterizing the vibration generated, in terms of frequency spectrum and intensity. Such information has been subsequently exploited to design a properly customized system for real-time vibration monitoring for health hazard assessment. A device has been thus developed taking advantage of wireless sensing and communicating technologies in order to allow in-field measurement during workers' operations. A final outdoor harvesting test has been performed to validate the system, collect the results and draw the conclusions. These activities are described in detail in the following sections.

\subsection{Preliminary vibration analysis}

The preliminary vibration analysis has been performed by means of traditional (wired) vibration assessment system available in the laboratory, in order to characterize the vibration and to identify the spectrum range of interest. The experimental tests have been carried out on a portable shaker mod. Cifarelli SC800 given in Fig. 1, whose technical features are given in Table 1.

Cifarelli SC800 is a hand held shaker commonly employed in olive harvesting operations. It is equipped with an anti-vibrating patented system on the handles that isolates them from the rod, from the engine and from the gear box. This system is designed with the aim of reducing the stresses transmitted, under operating conditions, to the user. The elastic elements interposed between the gripping means and the support body, compensate the reaction forces transmitted parallel to the drive axis of the movable shaft, allowing a prolonged use of the shaking device without causing heavy discomfort to the operator. Additionally, a particular kinematic mechanisms for the transmission of motion between the motor and the translating shaft minimizes both the vibrating stresses and the moments whose axis does not coincide with the axis of the movable shaft, thus making the support and use of the subject machine easier and less cumbersome. It should also be noted that the positioning of both elastic elements with axis parallel to the direction wherein the greater stresses are generated allows the best performance of the elements themselves. Such system also prevents the onset of additional forces with a direction not parallel to the elastic axis.

Particular attention was used during the fixing process of the accelerometers on the auxiliary and command handles, in order to have each axis oriented in the directions imposed by the provisions of UNI EN ISO 5349-1, 2001 (basicentric coordinate system): $y_{h}$ axis parallel to the axis of the handle; $x_{h}$ perpendicular to the axis of the handle oriented by the back towards the palm of the hand and, at last, the $z_{h}$ axis perpendicular to the plan formed by the two previous axis as shown in Fig. 2. For practical measurements, the orientation of the coordinate system may be defined with reference to an appropriate basicentric coordinate system originating in vibrating handle gripped by the hand.

The vibration tests have been performed inside the lab in simulated operating conditions with a fake branch constituted by a wooden bar with one end fixed to a solid structure and the other end free to shake.

Frequency analysis on laboratory tests was performed in the range of $0-1250 \mathrm{~Hz}$, and a sample FFT spectrum referred to the range $0-250 \mathrm{~Hz}$ of the vibrations on the $X$-axis of the main handle is given in Fig. 3. The spectrum shows a first harmonic

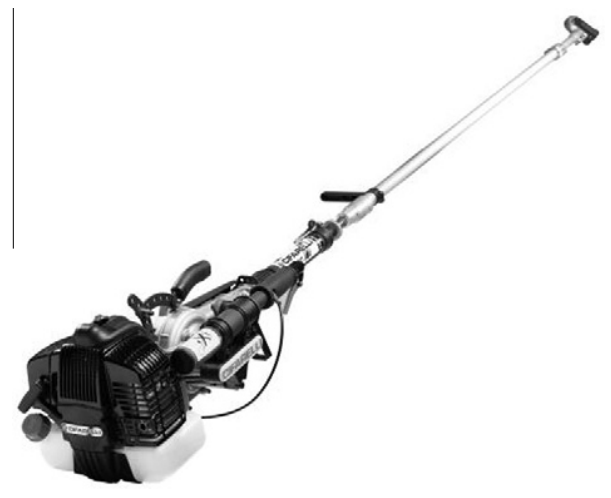

Fig. 1. Cifarelli SC800 hand held shaker tool. 
Table 1

Cifarelli SC800 technical features.

\begin{tabular}{ll}
\hline Engine name & Cifarelli C5 \\
\hline Engine displacement $\left(\mathrm{cm}^{3}\right)$ & 52 \\
Strokes $(\mathrm{n})$ & 2 \\
Cooling & Air \\
Tank capacity (lt) & 1.7 \\
Total weight (filled up) (kg) & 18.0 \\
Length except the bar (mm) & 1050 \\
Bar Length/total length ratio & 0.66 \\
Length of the bar (mm) & 2000 \\
Stroke of the bar (mm) & 60.2 \\
Hook width (mm) & 40.5
\end{tabular}

$15-25 \mathrm{~Hz}$, which corresponds to the rod oscillation at 1500 strokes/min (maximum), an additional harmonic occurring at approximately $130-140 \mathrm{~Hz}$ attributable to the engine vibration and some other minor harmonics in the range $200-250 \mathrm{~Hz}$ which are actually negligible for considering the ISO weighting curve.

\subsection{Vibration measurement system}

The measurement system briefly described below has been designed on the basis of the preliminary vibration analysis and taking into account the requirement of measuring the vibration levels affecting the hand-arm system of a workman in real operating conditions. Such conditions involve possible exposure to water, elevated temperatures, electrical interference, hazardous-area classifications, obstructions, physical location and distance.

The MEMS accelerometer is a device that converts a physical acceleration into an voltage output. The signal processor performs signal conditioning and pre-buffering for data transmission, and a workstation is finally used to display sensor data for real time monitoring and processing. The measuring chain contains the digital $x y z$ axis capacitive accelerometer, the I2C converter for converting I2C to UART and the XBee module for wireless transmission.

The described components are arranged in two separate assemblies (see Fig. 4): a wearable remote unit involving the sensors and the signal processor and the transmitting ZigBee unit, and a fixed station constituted by a computer and a ZigBee communication module for receiving data.

The device employed in our study is based on the ZigBee network which is the industrial standard for monitoring and control sensor networks defined by Motorola and conforming to the IEEE 802.15.4 standard. ZigBee networks are in fact self configuring and self healing, maximizing reliability and minimizing the cost of network deployment and maintenance. They provide transmission speeds of 20,40 and $250 \mathrm{~Kb} / \mathrm{s}$ over a range of $10-100 \mathrm{~m}$ and can be configured in star, mesh or peer-to-peer topologies. In addition ZigBee networks use considerably less power than all other networking technologies (Gutierrez et al., 2001; Zigbee Alliance, 2005).

The proposed application, however, presents some crucial differences compared with common configurations of current low

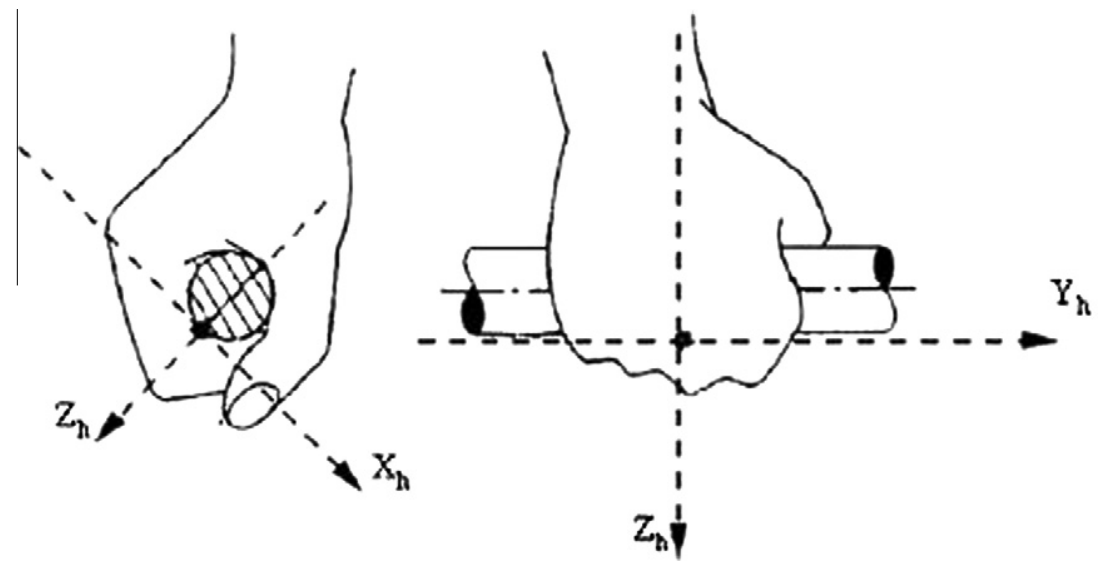

Fig. 2. Accelerometer position and directions.

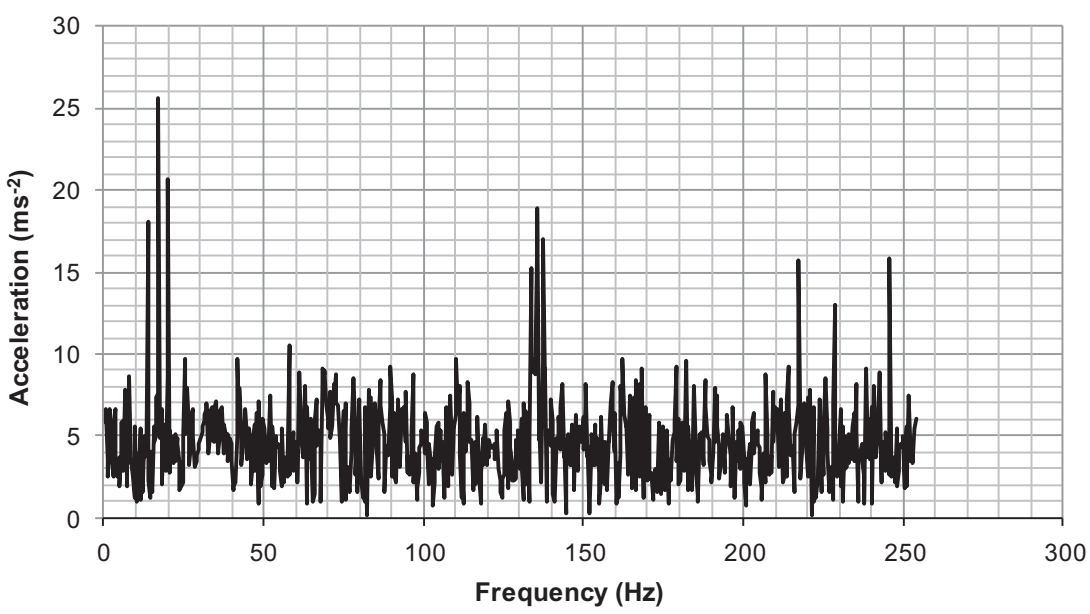

Fig. 3. Sample vibration spectrum (X axis) 


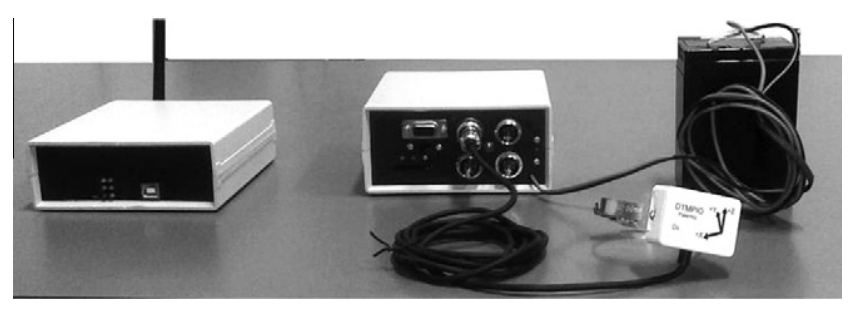

Fig. 4. Overview of the system developed.

power sensor networks. Most of the applications of wireless sensor networks in fact involve small data packets and intermittent or low periodic and low latency data and the related sensor nodes are consequently most of the time in a power saving sleep mode. This is not the case of real-time wireless vibration monitoring of multiple devices where bandwidth limitation may become a critical issue. In fact, having the ZigBee protocol 16-bit addresses it can theoretically connect up to 65532 devices, but in reality bandwidth is what limits the number of devices the network can have. It has been shown (Kohvakka et al., 2006) that the actual bandwidth for a ZigBee network is $157 \mathrm{~Kb} / \mathrm{s}$ after taking into account acknowledgements time, headers and inter-frame delays. Consequently, in order to monitor simultaneously a set of vibration sensing devices, the polling rate and data transmission features must be determined coherently with the available bandwidth. By properly regulating such parameters, the proposed architecture permits to manage a distributed sensor network, thus making it possible to remotely control the status of several workers simultaneously.

The wearable unit is attached to the operator's waist during its working activity, while the fixed station is placed nearby within the range of the ZigBee communication modules.

\subsection{Wearable sensor unit}

The wearable sensor unit has been designed taking into account that the unit must be lightweight, small and compact as possible in order not to hamper the operations and the movements of the worker. According to such specifications, the Freescale MMA7455 triaxial accelerometer device based on Microelectromechanical systems (MEMS) technology has been selected. This device is much cheaper thus making the whole system cost-effective for real-life applications.

MEMS-based sensors are a class of devices constituted by very small electrical and mechanical components on a single chip. MEMS capacitive accelerometers, in particular, have several advantages for numerous applications including low cost, high sensitivity, good response, noise performance, low temperature sensitivity, and low power dissipation, as documented by several researchers (Yazdi et al., 1998 and Bernstein et al., 1999). Due to such advantages, they are being employed in many safety systems to prevent and reduce health hazards. Mathie et al. (2004) and Chen (2005), for example, applied MEMS accelerometers to study human movements for fall detection purposes. Additionally, due to the versatility of MEMS sensors and their small size, they have been efficiently employed for monitoring vibrations in engines, mechanical equipments and facilities (Vogl et al., 2009) and in civil structures (Lynch et al., 2002). The possibility of employing such systems in vibration risk assessment is also a field of application recently approached and investigated by researchers (Wang et al., 2006; Koenig et al., 2008; Morello et al., 2010).

The proposed system is based on an accelerometer that has three selectable operating ranges: $\pm 2 \mathrm{~g}, \pm 4 \mathrm{~g}, \pm 8$ g. Each range provides different measurement sensitivity. In addition the MMA7455 accelerometer incorporates an on-chip FIFO (first-in/first-out) memory buffer that stores up to 32 sample sets of $X, Y$ and $Z$ data. This improves overall system power savings and response time by offloading functions from the host processor. Utilizing the FIFO buffer along with the other embedded functions allows the host processor to analyze only the required data while at the same time, protecting from the possibility of data loss achieving consumption savings that can range from 78 percent up to 96 percent or higher depending on conditions of the microcontroller and output data rates chosen. The main feature that makes the Freescale accelerometer suitable for in-field vibration testing is its extremely flexible performance/power consumption options through user-configurable sample rates (see Table 2). The rates can be adjusted to provide only the performance needed for specific functions, and each configuration corresponds with different current consumption.

The maximum sampling rate $(120 \mathrm{~Hz})$ is probably the most significant performance limit of the device selected, which is however appropriate for the application considered where the main harmonics are below $50 \mathrm{~Hz}$. The acceleration data collected by means of the previously described sensor are fed into a microcontroller and sampled via an analog digital converter (ADC). We selected the CUBLOC SC830 microcontroller, based on the Atmel ATmega128 processor for such purpose. The Atmel ATmega 128 is one of the most powerful 8bit micro controllers running at a frequency up to $16 \mathrm{MHz}$. Such device is designed for applications from display controls, in the telemetry sector, complex controlling tasks up to extending robot instructions. The microcontroller does simple processing on the data and sets the working mode of the accelerometer accordingly. Processed data are fed into an IEEE 802.15.4 wireless transceiver and sent to the data logger unit. For our design, the XBee 802.15.4 radio modem from MaxStream has been chosen as the wireless transceiver. It operates with a chip antenna up to $30 \mathrm{~m}$ indoor, under transparent mode with a simple connection with a microcontroller. The transmission range can be further increased to $90 \mathrm{~m}$ by using a whip antenna. The XBee module has a low maximum transmit power of $1 \mathrm{~mW}$ and a high receiver sensitivity of $-92 \mathrm{dBm}$. Finally the device involves a MMC (MultiMediaCard) reader/writer interfaced with CUBLOC SC830 via the SPI (Serial Port Interface).

Finally, as the amount of data gathered increases, the system requires solid Quality Assurance (QA) and Quality Control (QC) procedures in order to detect and purge potential erroneous data. Time variability test performed on each station by checking the variability during a certain period, are generally a standard procedure for such purpose. The following Fig. 5 shows a schematic of the system (a), and the device built (b). Fig. 6 shows the device worn by the operator.

\subsection{Data logger unit}

Data gathered by the wearable unit are transmitted to the data logger unit by means of a wireless communication channel. The front-end of the data logger unit is a wireless XBee transceiver which upon receiving the measurement data from the wireless interface, forwards the data directly to the workstation for processing by means of a software package. Gathered data are hence

Table 2

Polling frequencies and corresponding current consumption of the Freescale sensor.

\begin{tabular}{llll}
\hline Sample/s & Minimum & Typical & Maximum \\
\hline 1 & $38 \mu \mathrm{A}$ & $47 \mu \mathrm{A}$ & $62 \mu \mathrm{A}$ \\
2 & $37 \mu \mathrm{A}$ & $49 \mu \mathrm{A}$ & $60 \mu \mathrm{A}$ \\
4 & $48 \mu \mathrm{A}$ & $54 \mu \mathrm{A}$ & $83 \mu \mathrm{A}$ \\
8 & $59 \mu \mathrm{A}$ & $66 \mu \mathrm{A}$ & $86 \mu \mathrm{A}$ \\
16 & $70 \mu \mathrm{A}$ & $89 \mu \mathrm{A}$ & $100 \mu \mathrm{A}$ \\
32 & $85 \mu \mathrm{A}$ & $133 \mu \mathrm{A}$ & $171 \mu \mathrm{A}$ \\
64 & $170 \mu \mathrm{A}$ & $221 \mu \mathrm{A}$ & $262 \mu \mathrm{A}$ \\
120 & $224 \mu \mathrm{A}$ & $294 \mu \mathrm{A}$ & $350 \mu \mathrm{A}$ \\
\hline
\end{tabular}


processed for subsequent vibration analysis and health hazard assessment.

\subsection{Data analysis}

Acceleration data have been processed in real time in order to evaluate the health risk the worker is exposed to, according to the guidelines for measuring and evaluating human exposure given in ISO 5349-1 and ISO 5349-2, 2001. In the ISO 5349 standard recommendations, the most important quantity used to describe the magnitude of the vibrations transmitted to the operator's hands is the root-mean square (rms) frequency-weighted acceleration, expressed in $\mathrm{m} / \mathrm{s}^{2}$. According to such guidelines, the vibration spectrum must be extracted from the raw acceleration data by means of Fast Fourier Transformation (FFT), and analyzed in one-third-octave bands. Subsequently, in order to determine the effect of the vibrations on the human body the rms intensity in each band must be multiplicated by a proper weighting factor. It is in fact well known that human response to the hand-transmitted vibration varies enormously with frequency, and in particular, it is high at low frequency and greatly decreases with frequency (Reynolds et al., 1977; Broyde et al., 1989; Giacomin et al., 2004; Morioka and Griffin, 2006). ISO 5349 standard, in particular, recommends a weighting curve which shows a peak value for the weighting factor close to 1 , in the range between 8 and $16 \mathrm{~Hz}$, and a rapidly decreasing curve reaching values below 0.1 for frequencies above $150 \mathrm{~Hz}$. According to such weighing curve, acceleration values from one-third-octave band analysis can be used to obtain the frequency-weighted acceleration in each axis calculated as:

$a_{h w(x, y, z)}=\left[\sum_{j=1}^{n}\left(W_{j} \cdot a_{w, j}(x, y, z)\right)^{2}\right]^{1 / 2}$

where $a_{w, j}$ is the acceleration measured in the one-third octave band in $\mathrm{m} / \mathrm{s}^{2}$, and $W_{j}$ is the weighting factor for the one-thirdoctave band. The evaluation of vibration exposure in accordance with ISO 5349 is finally based on a quantity that combines all the three axes. This is the vibration total value $a_{h w}$ or weighted acceleration sum (WAS) and it is defined as the root-mean-square of the three component values:

$a_{h w}=\sqrt{a_{h w(x)}^{2}+a_{h w(y)}^{2}+a_{h w(z)}^{2}}$

where $a_{h w x}, a_{h w y}, a_{h w z}$ are frequency-weighted acceleration values for the single axes. The vibration exposure depends on the magnitude of

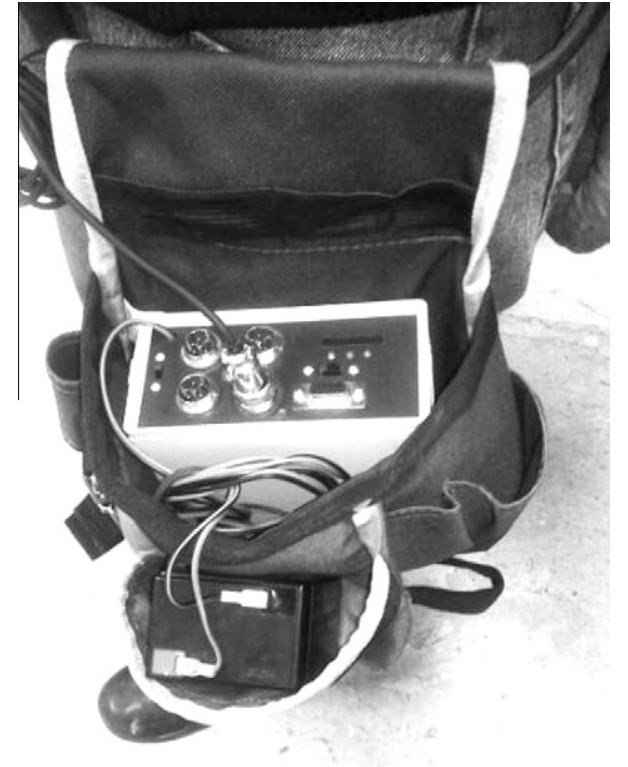

Fig. 6. The wearable unit attached to the operator's waist.

the vibration total value and on the duration of the exposure. Daily exposure duration is the total time for which the hands are exposed to vibrations during the working day. The daily vibration exposure shall be expressed in terms of the 8-h energy-equivalent acceleration or frequency-weighted vibration total value:

$A(8)=a_{h w} \sqrt{\frac{T}{T_{0}}}$

where $T$ is the total daily duration of the exposure in seconds, and $T_{0}$ is the reference duration of $8 \mathrm{~h}(28800 \mathrm{~s})$.

\section{Results and discussion}

The results from the preliminary analysis performed in the laboratory showed that the $X$-axis of the main handle was characterized by the most significant vibrations levels, although the same considerations on the spectrum analysis can be done for all the other axes and measures. The first harmonic appears at $15-25 \mathrm{~Hz}$, as the main vibration component, and corresponds to the rod oscillation occurring at maximum 1500 strokes/min $(25 \mathrm{~Hz})$, as declared
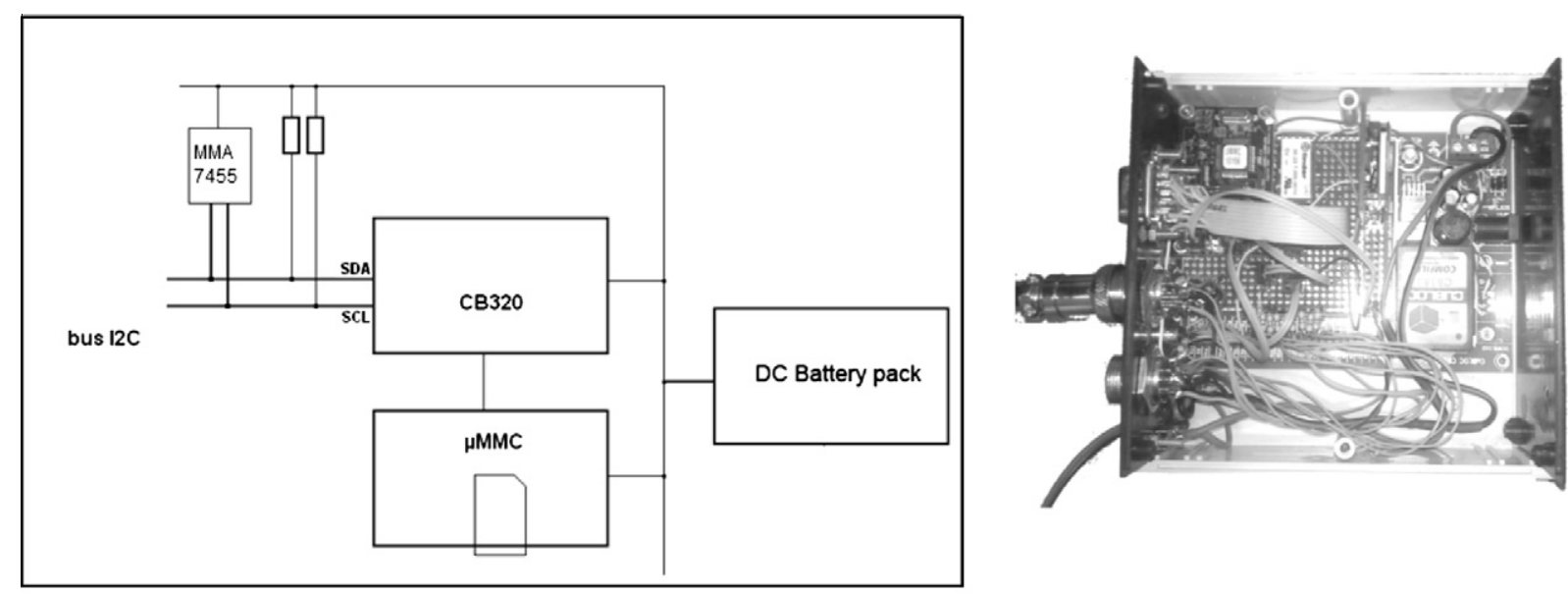

(a)

Fig. 5. Schematics (a) and picture (b) of the device developed 
Left Hand
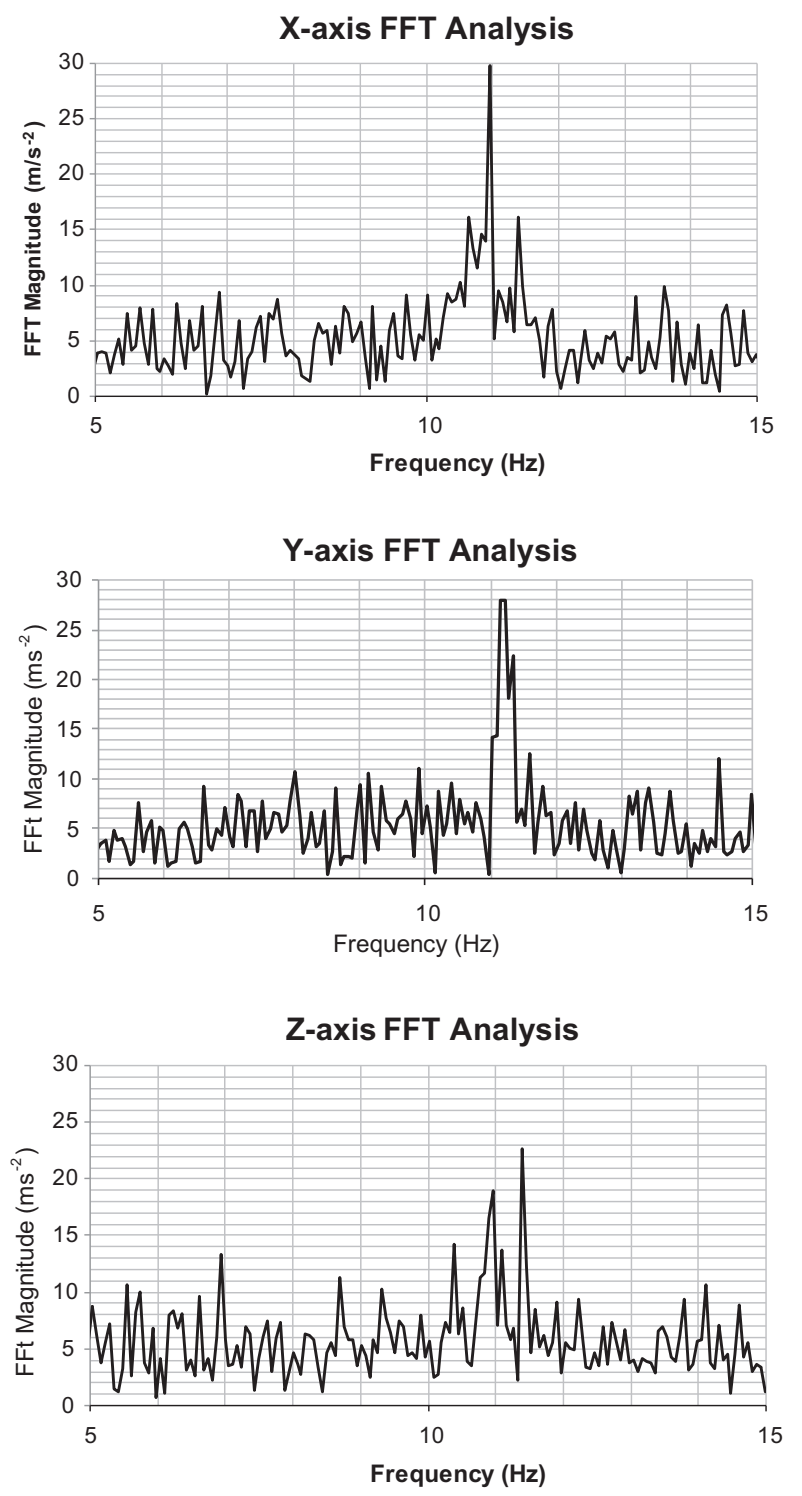

Right Hand
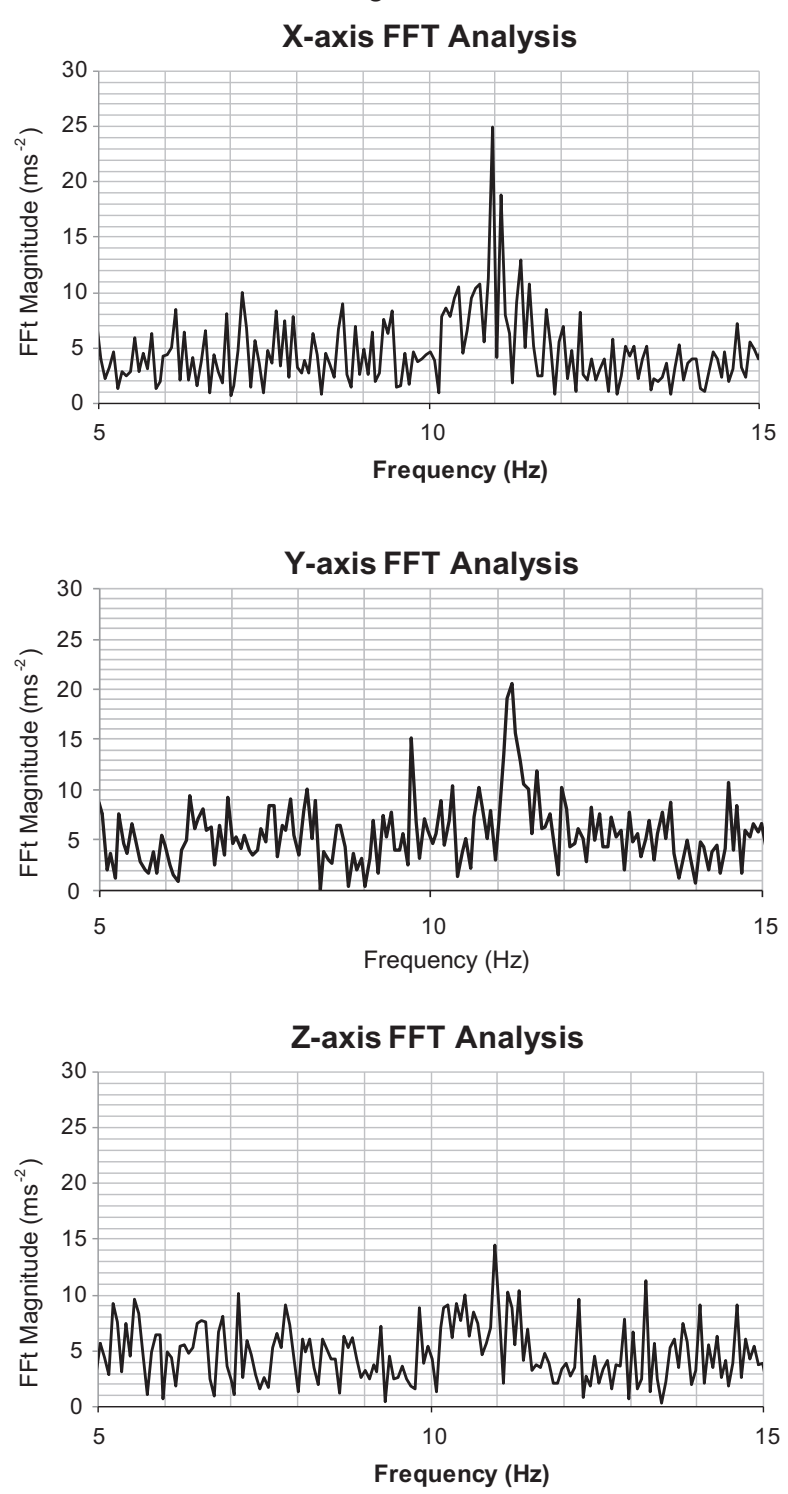

Fig. 7. Vibration spectra in $x, y$ and $z$ directions for both hands.

by the manufacturer. An additional harmonic occurs at approximately $130-140 \mathrm{~Hz}$ which is most likely attributable to the engine vibration as in fact the maximum operating speed of the engine is approx. $8100 \mathrm{rpm}$. Finally, the spectrum shows some other appreciable harmonics in the range $200-250 \mathrm{~Hz}$, which appear mainly in $X$ and $Z$ directions, and less significant in the $Y$ axis. The full-spectrum analysis allowed to conclude that the vibration range where the most risky vibration phenomena occur is $0-50 \mathrm{~Hz}$. It has also been considered the contribution of vibrations over $100 \mathrm{~Hz}$ are actually negligible for safety risk according to the ISO weighting curve. The results obtained are coherent with similar results obtained by other researchers (Pascuzzi et al., 2009).

Experimental tests in real operating conditions, subsequently carried out involved the real time acquisition and processing of the acceleration data gathered by means of the monitoring device developed. Each test consisted in 3 trials of $40 \mathrm{~s}$ with a polling frequency of $120 \mathrm{~Hz}$. Raw acceleration data are represented by 8 bit integer values thus generating a data-stream of $120 \times 8$ bits plus a 32 bit timestamp per second, for each axis. Total data communication requirement hence accounts for an overall of $4800 \mathrm{bit} / \mathrm{s}$ of bandwidth. Such measurement is repeated in $X, Y$ and $Z$ axis in each hand thus generating 6 data streams with an overall $30 \mathrm{~kb} / \mathrm{s}$ bandwidth approximately required. Additionally, before each test, a calibration step was carried out by placing the MMA7455 sensor flat with the $z$ axis pointing up so that the $X$ and $Y$ axis will initially read zero and the $Z$ axis will read $1 \mathrm{~g}$. The $0 \mathrm{~g}$ offset has thus been calibrated using the assigned registers and g-Select register was positioned to $8 \mathrm{~g}$. The data series thus obtained were analyzed as described in the previous paragraph.

The FFT spectrum, for the vibrations along the $X, Y$ and $Z$ axis measured in the experimental tests for both hands show that vibration acceleration in the $X$-axis, is more prominent than the other two directions, and $Z$ axis is generally less affected by vibration phenomena. In particular for the left hand, the peak vibration acceleration was approximately $30 \mathrm{~m} / \mathrm{s}^{2}$ on the $x$ axis, at the frequency of $11-14 \mathrm{~Hz}$. The same harmonic can be distinguished in $y$ and $z$ directions. Different considerations can be done for the right hand, where again the FFT spectra show the same peak in the $x$ axis, and a lower vibration level on the other two axis. In particular vibration on the $z$ axis of the right hand is significantly 

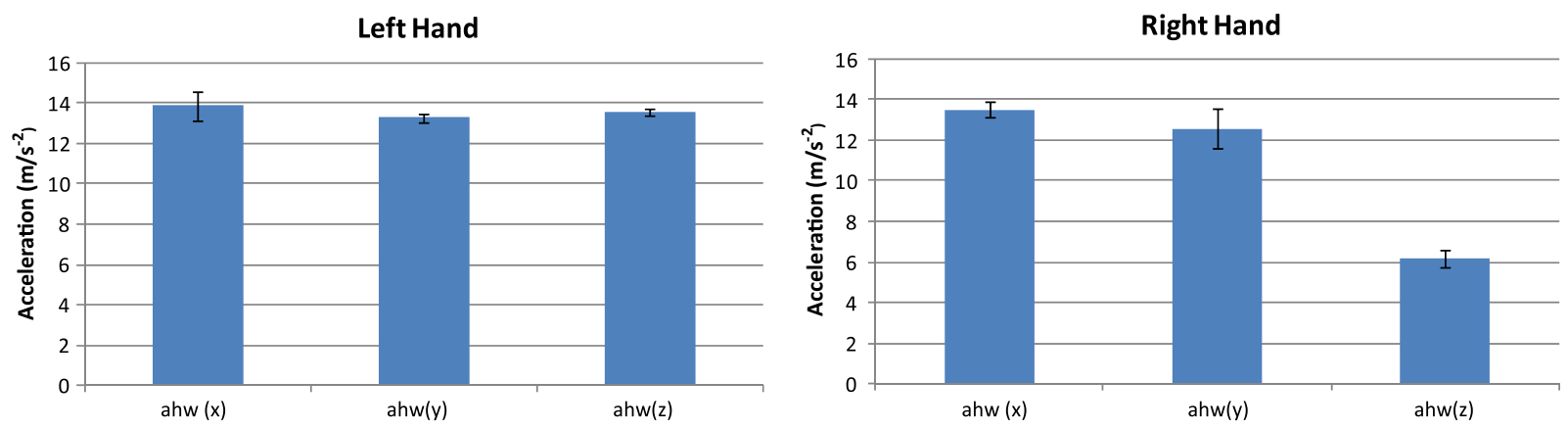

Fig. 8. $a_{h w}(x, y, z$, ) values for both hands. The error bars represent the spread between maximum and minimum measurements.

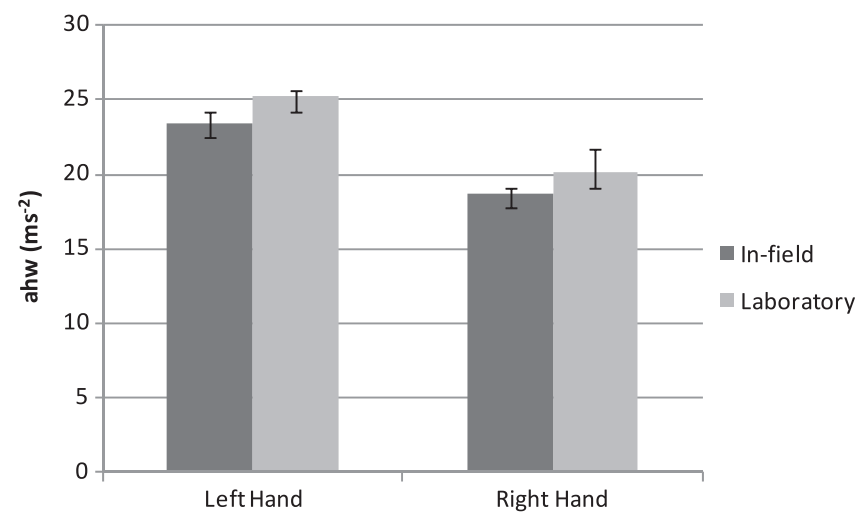

Fig. 9. Comparison of $a_{h w}$ values for both hands in laboratory and in-field tests.

smaller than all others. These considerations hold for all the data analyzed, as the sample spectrum given in the following Fig. 7 reveals. A deeper statistical analysis would be required to assess the performance of the tools analyzed: this however falls outside the scope of the paper, which on the contrary focuses on the measurement system.

The resulting weighted vibration level for each axis, calculated according to Eq. (1) is given in the following Fig. 8.

Finally the comparison with laboratory tests shows the approximation of the wireless vibration device with more precise laboratory instruments. The comparison given in Fig. 9 shows that although the experimental tests generally lead to a underestimation of the vibration exposure, the error is generally lower than $10 \%$, thus demonstrating a fairly acceptable compromise between performance and ease of operation of the monitoring system.

The results given above suggest that the system designed is suitable for the purpose of monitoring several workers simultaneously via a wireless communication system, and the approximation which derives from the performance limits of the technology employed, is an acceptable compromise in order to maintain the economic affordability of the system, since a measurement system perfectly compliant with the standards would be technically impossible or extremely expensive. Clearly these considerations are referred to the tools analyzed in the experimental tests and to the vibration frequencies they generate, as in fact the system design is strictly linked to the applicative scenario considered. The extension to other tools and applications involves to update the system design and the technological features, which might significantly influence the costs thus preventing the achievement of a feasible compromise.

\section{Conclusions}

Vibrations are a well known potential cause of health diseases and therefore constitute a main concern for the safety of workers in a large number of activities. In agriculture, in particular, the diffusion of mechanization has dramatically increased the risk exposure for workers thus raising the risk profile of the entire sector towards vibration. National and international directives aimed at preventing the possible health treaties are based upon the measurement of the vibration level the workers are exposed to during their activities. Measuring vibrations however is technically difficult, because it involves precise and expensive laboratory instruments which can hardly be employed in real operating conditions, and it requires complex computational effort and timeconsuming procedures. In such context recent emerging technologies such as MEMS accelerometers and wireless networking allow a revolutionary approach to safety management, by promoting the development of cheap devices for real time monitoring and risk assessment.

This research demonstrates that technologies are mature enough to develop cheap and inexpensive devices for vibration risk assessment. In particular, the research involved the development of a novel device for assessing the health risk caused by handarm vibrations in real worker operating conditions. The device developed can easily be integrated into workers' standard equipment employed in outdoor operations, since it is involves a lightweight wearable device with on board power supply and wireless communication module. The experimental field tests even in their preliminary stage seem to confirm the effectiveness of the research proposed in assessing workers' risks exposure during harvesting operations, although a deeper analysis would probably be appropriate. The results obtained show how a simple and cheap hardware can lead to a fairly precise assessment of the risk exposure, thus providing significant information to the management in defining safe work-shifts and improving the resource scheduling and allocation. The study allows for methodological considerations, susceptible to be integrated by further investigations, and proposes new sceneries in intelligent worker safety management systems, where workers' shifts and activities are organized in a more responsible way coherently with safety principles and directives, by means of decision support systems based on real time data acquisition. In addition the vibration data might also be exploited for the management of maintenance operations based on the health status of the machines, while the integration with other sensing devices might allow for further developments in the assessment of workers' wellness.

It is hence possible to foresee future developments which might significantly modify the current approach to safety risk management, including for example intelligent protection devices, integrated with workers' equipment and clothing such as intelligent gloves, or jackets equipped with sensor and wireless transmission devices, etc. Such system may be crucial for the development of safety management systems able to monitor the risk exposure of several works simultaneously in real operating conditions. 


\section{Acknowledgements}

The authors are grateful to Mr. Saverio Pantaleone and to Leonardo Engineering and Research s.r.l. for supporting the device development process.

\section{References}

Aldien, Y., Marcotte, P., Rakheja, S., Boileau, P.E., 2006. Influence of hand forces and handle size on power absorption of the human hand-arm exposed to $z$-axis vibration. J. Sound Vib. 290, 1015-1039.

Bernstein, J., Miller, R., Kelley, W., Ward, P., 1999. Low-noise MEMS vibration sensor for geophysical applications. J. Micro. Elect. Mech. Syst. 8, 433-438.

Broyde, M., Donati, P., Bitsch, J., 1989. Subjective assessment of the vibration discomfort produced by different hand held machines. J. Low Freq. Noise Vib. 8 (2), 50-61.

Burström, L., 1997. The influence of biodynamic factors on the mechanical impedance of the hand and arm. Int. Arch. Occup. Environ. Health. 69, 437-446.

Chen, W., 2005. Mobile Phone-based wearable vital signs monitoring system, Conference on Computer and Information Technology, CIT05. 950-955.

Dewangan, K.N., Tewari, V.K., 2008. Characteristics of hand-transmitted vibration of a hand tractor used in three operational modes. Int. J. Ind. Erg. 100, 535-546.

Gerhardsson, L., Balogh, I., Hambert, P.A., Hjortsberg, U., Karlsson, J.E., 2005 Vascular and nerve damage in workers exposed to vibrating tools: the importance of objective measurements of exposure time. J. Appl. Ergon. 36, $55-60$.

Giacomin, J., Shayaa, M.S., Dormegnie, E., Richard, L., 2004. Frequency weighting for the evaluation of steering wheel rotational vibration. Int. J. Ind. Erg. 33, 527541.

Gil, J., Blanco, G.L., Agüera, J. 2001. Optimization of the design and use of shaker machines for mechanically harvesting of the olive trees in Spain. ASAE Paper $n$. 01-1096.

Gutierrez, J.A., Naeve, M., Callaway, E., Bourgeois, M., Mitter, V., Heile, B., 2001. IEEE 802.15.4: a developing standard for low-power, low-cost wireless personal area networks. IEEE Network 15 (5), 12-19.

Horváth, E., Sitkei, G., 2001. Energy consumption of selected tree shakers under different operational conditions. J. Agr. Eng. Res. 80 (2), 191-199.

ISO 5349-1, 2001. Mechanical Vibration - Guidelines for the Measurement and Assessment of Human Exposure to Hand-transmitted Vibration. Part - 1: General Requirements. International Standard Organization, Geneva.
ISO 5349-2, 2001. Mechanical Vibration - Guidelines for the Measurement and Assessment of Human Exposure to Hand-transmitted Vibration. Part - 2: Practical Guidance for Measurement at the Workplace. International Standard Organization, Geneva.

Koenig, D., Chiaramonte, M.S., Balbinot, A., 2008. Wireless network for measurement of whole-body vibration. Sensors 8, 3067-3081.

Kohvakka, M., Kuorilehto, M., Hännikäinen, M., Hämäläinen, T.D., Performance analysis of IEEE 802.15.4 and ZigBee for large-scale wireless sensor network applications, 3rd ACM international Workshop on Performance Evaluation of Wireless Ad Hoc, Sensor and Ubiquitous Networks. Torremolinos, Spain, October 2006.

Lynch, J.P., Law, K.H., Kiremidjian, A.S., Carryer, E., 2002. Validation of a wireless modular monitoring system for structures, 9th Annual Int. Symp. on Smart Structures and Materials. 124-135.

Mathie, M.J., Coster, A.C.F., Lovell, N.H., Celler, B.G., 2004. Accelerometry: providing an integrated, practical method for long-term, ambulatory monitoring of human movement. Physiol. Meas. 25, 1-20.

Morello, R., De Capua, C., Meduri, A., 2010. A wireless measurement system for estimation of human exposure to vibration during the use of handheld percussion machines IEEE transactions on instrumentation and measurement 59 (10), 2513-2521.

Morioka, M., Griffin, M.J., 2006. Magnitude-dependence of equivalent comfort contours for fore-and-aft, lateral and vertical hand-transmitted vibration. J. Sound Vib. 295, 633-648.

Pascuzzi S., Santoro F., and Panaro V.N., 2009. Investigation of workers' exposures to vibrations produced by portable shakers, Agricultural Engineering International: the CIGR Ejournal. XI.

Reynolds, D.D., Standlee, K.G., Angevine, E.N., 1977. Hand-arm vibration. Part 3: subjective responses characteristics of individuals to hand induced vibration. J. Sound Vib. 51 (2), 267-281.

Vogl, A., Dag, T., Wang, P.S., Bakke, T., Maaike, M.V., Taklo, M.M.V., Thomson, A., Balgård, L., 2009. Design, process and characterisation of a high-performance vibration sensor for wireless condition monitoring. Sensors and actuators a: physical 153 (2), 155-161.

Wang, N., Zhang, N., Wang, M., 2006. Wireless sensors in agriculture and food industry-recent development and future perspective. Comput. Electron. Agric. 50, 1-14.

Yazdi, N., Ayazi, F., Najafi, K., 1998. Micromachined inertial sensors. Proc. IEEE. 86, 1640-1659.

Zigbee Alliance, 2005 Zigbee specification. Technical Report Document 053474r06, version 1.0, Zigbee Alliance. 